\title{
Intranasal Eruption of an Impacted Supernumerary - A Case Report
}

\author{
Eman Bin Saleh¹, Manju Philip² \\ 1, 2 Department of Maxillofacial Surgery, College of Dentistry, King Saud University, KSA.
}

\section{INTRODUCTION}

Supernumeraries erupting in ectopic regions are one of the commonly found anomalies of orofacial region. ${ }^{1}$ The mesiodens, being located between the maxillary central incisors is the most common supernumerary tooth.2,3 But impacted mesiodens erupting into nasal cavity is a rare finding. We are detailing an instance of an inverted impacted mesiodens in the nasopalatine canal of an adult patient with encroachment on the nasal floor diagnosed as an incidental finding in panoramic radiograph. Intranasal tooth in adults is reported rare in the literature. Although surgical removal is advised generally on diagnosis of impacted mesiodens, we stress the importance of periodic monitoring of such cases which adds significantly to the patient wellbeing, function and aesthetics. There are few published case reports and most of the cases reported in the literature are in children which prompt for immediate removal upon its recognition. A supernumerary tooth that erupts ectopically into the nasal cavity is referred to as an intranasal tooth. Its occurrence rate is low with only 0.1 to $1 \%$ people being affected from the community. ${ }^{4}$ The aetiology of intranasal tooth stays undefined. Supernumerary teeth may develop from the region of the nasal cavity as a result of a damage caused by an injury, rhinogenic maxillary sinusitis, a dental disease, oral blisters, obstructed path of dental eruption, and disruption of developmental tissue in the palatine gap. ${ }^{4}$ The potential complications that can arise due to nasal eruption of tooth are perforation of nasal septum, naso-oral fistula and aspergillosis 4 . The probable dental complications include midline diastema $(17.6 \%)$, late eruption of the permanent incisors (38.8\%), axial rotation of the permanent incisors (16.4\%), root resorption of the neighbouring teeth $(4.7 \%),{ }^{5}$ cyst formation, ${ }^{6}$ and infection. ${ }^{1}$ A team approach with inclusion of disciplines in medicine and dentistry is necessary for planning and execution of an appropriate treatment method in order to prevent complications related to inadvertent surgical entry into vital structures.

\section{PRESENTATION Of CASE}

A female patient in her early thirties reported to Comprehensive Clinical Dentistry department for dental treatment. She had no history of systemic medical conditions. Her physical status was ASA 1 according to ASA physical status classification. She had history of extraction of an extra tooth located between lower left premolars. Her family history of additional teeth and associated syndromes was non-significant.
Corresponding Author: Dr. Manju Philip, MDS, FDSRCS, FDSRCPS, Maxillofacial Surgery, College of Dentistry, King Saud University, KSA.

E-mail: drmanjuphilip@gmail.com

DOI: $10.14260 / \mathrm{jemds} / 2021 / 514$

How to Cite This Article: Saleh EB, Philip M. Intranasal eruption of an impacted supernumerary - a case report. J Evolution Med Dent Sci 2021;10(31): 2514-2516, DOI:

$10.14260 / \mathrm{jemds} / 2021 / 514$

Submission 10-11-2020,

Peer Review 07-07-2021,

Acceptance 13-07-2021,

Published 02-08-2021.

Copyright (c) 2021 Eman Bin Saleh et al. This is an open access article distributed under Creative Commons Attribution License [Attribution 4.0 International (CC BY 4.0)] 
Examination revealed an impacted permanent canine \#13 and retained primary canine. Cone beam computed tomography (CBCT) was taken for additional investigation and as part of comprehensive treatment plan including possibility of orthodontic treatment to facilitate eruption of impacted canine. CBCT has shown upper right permanent canine (\#13), impacted and located in the mid alveolus, causing resorption in relation to deciduous canine (\# 53). This tooth was rotated with the buccal surface towards the distal and palatal towards the mesial side and the root formation was complete. The crown of the impacted canine was in close approximation to the apical third of the root of lateral incisor leading to mild root resorption. Incidental findings revealed an impacted supernumerary located palatally between the central incisors, with inverted crown and the tip of crown piercing the nasal cavity. Also, it was in approximation and within the nasopalatine canal. [Figure 1]

Otolaryngology and maxillofacial surgery consultations were sought for further management of tooth erupting into the nasal floor. The mesiodens was encroaching the right nasal floor impinging on nasal mucosa without oral communication as shown in [Figure 2]
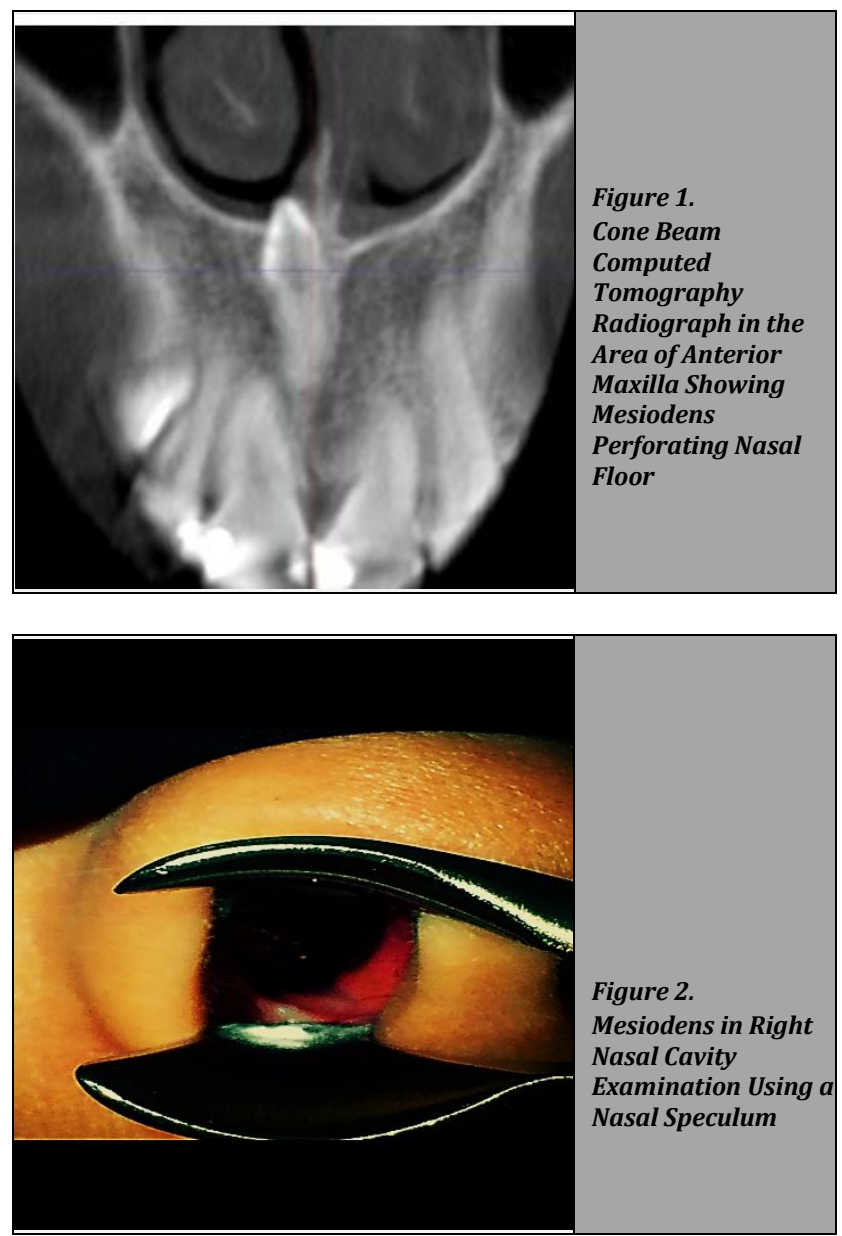

\section{DISCUSSION}

Regarding the impacted canine (\#13), orthodontic extrusion was advised on completion of the comprehensive dental plan. As for the impacted supernumerary tooth, both otorhinolaryngology and maxillofacial surgery departments recommend doing close follow up as the site was asymptomatic and any surgical attempt to remove could lead to displacement into tissue spaces, oronasal communication or fistula, nasal regurgitation, airway problems whistling, and neurosensory disturbances like paraesthesia.

Mesiodens may be found erupted or as impacted, single or as multiples (mesiodentes), unilateral or as bilateral.7 The prevalence of mesiodens in an inverted manner makes to approximately $9-67 \%$ of all reported cases. ${ }^{3}$ The location of impacted mesiodens in close approximation with the nasopalatine canal and perforating the nasal mucosa was occasionally reported in the literature. As the previously published literature were mostly in paediatric population, the present case report is exclusive in nature indicating the presence of mesiodens in nasopalatine canal and perforating nasal mucosa does not always necessitate surgical intervention and can remain asymptomatic for an adult patient. Of all the supernumerary teeth, 48 to $52 \%$ is represented by the mesiodens ${ }^{1}$. Impacted mesiodens constitutes $75 \%$ of which $6 \%$ are in a labial position, $80 \%$ occur palatally and $14 \%$ found between the maxillary central incisor's roots. ${ }^{1}$ Also teeth were found in a vertical position in $55.2 \%$ followed by inverted and horizontal positions in 37.6 $\%$ cases and $7 \%$ cases respectively.5,6 In the present report, the mesiodens was having conical roots, oriented palatally and crown perforating the right nostril.

The literature reports three theories to explain the cause of mesiodens. ${ }^{1,2}$ The first one was a phylogenetic theory. The second theory known as dichotomy states that the tooth bud is split into two teeth, one of which is the mesiodens. The third theory suggesting hyperactivity of the dental lamina was widely accepted. ${ }^{8}$ Mesiodens occur more in males with a malefemale ratio of $2: 1.1,8,9$ Cone beam computed tomography (CBCT) is an essential investigation that should be included in the management of impacted teeth giving a three-dimensional picture of the orofacial region in a low economical as well as dose efficient manner ${ }^{8}$ with high resolution of images. It can help in reducing surgical trauma to the adjacent vital tissues ${ }^{8}$. Due to the radiological unclear maxillary midline region, they can go unnoticed by the classic radiographic techniques generally used by the dental practitioner. ${ }^{9}$

The timing of surgical removal of mesiodens has two alternatives. First, it can be removed as soon as it is diagnosed, second is to delay until the root formation of adjacent teeth is completed in order to prevent damage to their apices.1,8 Periodic radiographic follow up is indicated even if they remain asymptomatic without causing interference with orthodontic tooth movement. ${ }^{4}$ Here we chose periodic monitoring of the case as it remained asymptomatic and also considering the chances of surgical entry into nasal floor causing long term complications. During the 2 year follow up period, our patient was informed of the possible complications that can arise due to infection and cystic changes in the region, but none was reported.

Mesiodens are reported in the literature associated with genetic abnormalities like cleidocranial dysostosis, cleft lip and palate, Gardner's syndrome and chondroectodermal dysplasia. $1,2,3,8$ Also the surgical management with secondary alveolar bone grafting and implant placement in cleft palate patients, can be compromised due to the presence of mesiodens. 


\section{CONCLUSIONS}

This case report highlights the importance of multidisciplinary management and shows their great role in diagnosing cases and improve the quality of social life and health of the patients. As most of the cases of impacted mesiodens reported previously were in children which necessitated surgical intervention without delay, this case report advocates for periodic follow up if asymptomatic, in adult patients.

Financial or other competing interests: None.

Disclosure forms provided by the authors are available with the full text of this article at jemds.com.

\section{REFERENCES}

[1] Jafri SAH, Pannu PK, Galhotra V, et al. Management of inverted impacted mesiodens, associated with a partially erupted supplemental tooth-a case report. Indian Journal of Dentistry 2011;2(2):40-3.

[2] Patil S, Maheshwari S. Prevalence of impacted and supernumerary teeth in the North Indian population. J Clin Exp Dent 2014;6(2):e116-20.
[3] Anusha RL, Halim N, Kalkur C, et al. Inverted impacted mesiodens-an incidental radiographic finding. IP International Journal of Maxillofacial Imaging 2017;3(4):126-7.

[4] Prasad RG, Nair PP, Gharote H, et al. Intranasal tooth-an ectopic eruption of mesiodens in nasal cavity: a case report and review. Journal of Indian Academy of Oral Medicine and Radiology 2011;23(3):252-5.

[5] Gunduz K, Celenk P, Zengin Z, et al. Mesiodens: a radiographic study in children. J Oral Sci 2008;50(3):28791.

[6] Ghogre P, Singh VD. Management of an impacted inverted mesiodens associated with a large circumferential type of dentigerous cyst: a rare case report with one year followup. Int Journal of Case Reports and Images 2014:5(1):805.

[7] Gharote HP, Nair PP, Thomas S, et al. Nonsyndromic double mesiodentes--hidden lambs among normal flock! BMJ Case Rep 2011;2011:bcr0720114420.

[8] Omami M, Chokri A, Hentati H, et al. Cone-beam computed tomography exploration and surgical management of palatal, inverted and impacted mesiodens. Contemp Clin Dent 2015;6(Suppl 1):S289-93.

[9] Aoun G, Nasseh I. Mesiodens within the nasopalatine canal: an exceptional entity. Clin Pract 2016;6(4):903. 